OPEN ACCESS

Edited by:

Andre Longtin,

University of Ottawa, Canada

Reviewed by:

Takuma Tanaka

Shiga University, Japan

Gregor Kovacic

Rensselaer Polytechnic Institute, USA

*Correspondence:

Lei Wang

wanglei_nc@163.com

Yanjun Zeng

yjzeng@bjut.edu.cn

Received: 20 April 2016 Accepted: 09 September 2016 Published: 23 September 2016

Citation:

Wang L, Qiu Y-H and Zeng Y (2016) Coding Properties of Three Intrinsically Distinct Retinal Ganglion Cells under Periodic Stimuli: A Computational

Front. Comput. Neurosci. 10:102. doi: 10.3389/fncom.2016.00102

\section{Coding Properties of Three Intrinsically Distinct Retinal Ganglion Cells under Periodic Stimuli: A Computational Study}

\author{
Lei Wang ${ }^{1 *}$, Yi-Hong Qiu ${ }^{2}$ and Yanjun Zeng ${ }^{3 *}$ \\ ${ }^{1}$ Neuroscience and Intelligent Media Institute, Communication University of China, Beijing, China, ${ }^{2}$ School of Biomedical \\ Engineering, Shanghai Jiao Tong University, Shanghai, China, ${ }^{3}$ Biomedical Engineering Center, Beijing University of \\ Technology, Beijing, China
}

As the sole output neurons in the retina, ganglion cells play significant roles in transforming visual information into spike trains, and then transmitting them to the higher visual centers. However, coding strategies that retinal ganglion cells (RGCs) adopt to accomplish these processes are not completely clear yet. To clarify these issues, we investigate the coding properties of three types of RGCs (repetitive spiking, tonic firing, and phasic firing) by two different measures (spike-rate and spike-latency). Model results show that for periodic stimuli, repetitive spiking RGC and tonic RGC exhibit similar spike-rate patterns. Their spike- rates decrease gradually with increased stimulus frequency, moreover, variation of stimulus amplitude would change the two RGCs' spike-rate patterns. For phasic RGC, it activates strongly at medium levels of frequency when the stimulus amplitude is low. While if high stimulus amplitude is applied, phasic RGC switches to respond strongly at low frequencies. These results suggest that stimulus amplitude is a prominent factor in regulating RGCs in encoding periodic signals. Similar conclusions can be drawn when analyzes spike-latency patterns of the three RGCs. More importantly, the above phenomena can be accurately reproduced by Hodgkin's three classes of neurons, indicating that RGCs can perform the typical three classes of firing dynamics, depending on the distinctions of ion channel densities. Consequently, model results from the three RGCs may be not specific, but can also applicable to neurons in other brain regions which exhibit part(s) or all of the Hodgkin's three excitabilities.

Keywords: retinal ganglion cell, morris-lecar neuron, spike-rate, spike-latency, periodic stimuli

\section{INTRODUCTION}

In the vertebrate retinas, ganglion cells are the principal neurons, through which visual information is effectively processed and then reliably transmitted to the lateral geniculate nucleus (LGN) and the visual cortex (Kandel et al., 2000). Thus, the capability of retinal ganglion cells (RGCs) in encoding incoming stimulus becomes extremely important. Previous studies suggested that RGCs can be classified into several different types based on their response patterns, e.g., repetitive spiking (Fohlmeister and Miller, 1997; Margolis and Detwiler, 2007; Fohlmeister et al., 2010), tonic firing (Wang et al., 1997; Tsai et al., 2011), phasic firing (Kawai and Sterling, 2002; Tabata and Kano, 2002), 
and adapting activity (Kim and Rieke, 2003; Li et al., 2012; Xiao et al., 2013a,b). These different firing behaviors may manifest different coding manners that RGCs adopt in processing neural signals.

It has been accepted that neural information can be encoded in a variety of manners, among which rate coding and time coding are the two most commonly used (Dayan and Abbott, 2001). Generally, rate coding refers to the number of spikes within a time window or cycle (Descalzo et al., 2005; van Brederode and Berger, 2008), while time coding refers to the first-spike latency of neural spikes (Chase and Young, 2007) or regularity or sparseness of population spike trains (Mainen and Sejnowski, 1995; Zhang et al., 2010). Several reports have revealed that RGCs can encode incoming signals effectively using these two strategies (van Rullen and Thorpe, 2001; Gollisch and Meister, 2008; Gütig et al., 2013). However, response patterns that RGCs produce under periodic stimuli are not completely clear yet, which need to be figured out.

Previously, Hodgkin classified neurons into three basic classes based on their firing frequency vs. stimulus intensity $(f-I)$ curves (Hodgkin, 1948). Generally, Class I neurons fire slowly in response to weak stimulus, and display a continuous $f-I$ curve; while class II neurons exhibit a discontinuous $f-I$ curve for their inability to produce spikes below certain threshold intensities. Class III neurons, however, fail to spike repetitively, and typically spike only once at the onset of stimulus (Izhikevich, 2007; Prescott et al., 2008a; Wang et al., 2013). Functional roles of the three classes of neurons have been extensively investigated during the past decades (Marella and Ermentrout, 2008; Bogaard et al., 2009; Fink et al., 2011). For instance, it has been proposed that class I neurons act as integrators or coincidence detectors, while class II neurons act as resonators (Izhikevich, 2000). For time-varying inputs, class III neurons serve as slope detectors or band-pass filters (Gai et al., 2010).

In this study, we firstly investigate how RGCs react to periodic stimuli through spike-rate coding and spike-latency coding. Ionic models which can characterize the electrophysiological properties of three RGCs (repetitive spiking, tonic firing, and phasic firing) are introduced. Simulation results demonstrate that diverse response patterns can be observed in RGCs, not only within the same types, but also among different types. In addition, stimulus amplitude plays a conspicuous role in transforming the response patterns from one type to another. Finally, we show that RGCs can exhibit all the Hodgkin's three classes of firing dynamics by only adjusting the conductances of several ion channels.

\section{MODELS AND METHODS}

\section{RGC Model}

Ionic model of RGCs with repetitive spiking was adopted from Fohlmeister and Miller (1997). The model had only onecompartment, which represents the soma, with five voltagegated ion channels and a leak channel $\left(I_{L}\right)$ distributed on the soma. The five ion channels were: inactivating sodium $\left(I_{\mathrm{Na}}\right)$, delayed-rectifier potassium $\left(I_{K}\right)$, calcium $\left(I_{C a}\right)$, A-type potassium $\left(I_{A}\right)$, and calcium-activated potassium $\left(I_{K C a}\right)$ channels. Later, to simulate the tonic and phasic firings in RGCs, a modified
RGC model was introduced (Wang et al., 2014). The modified model retained the five voltage-gated ion channels, but revised the inactivating sodium channel by adding two slow variables. Numerical results showed that the model can well reproduce the tonic and phasic firing behaviors by adjusting several parameters. Adapting activity is another typical firing behavior observed in RGCs, due to the lack of proper ion channels and corresponding neuron models, we did not consider adapting activity in the present study.

Mathematical description in Equation (1) represents the common model of RGCs (Fohlmeister and Miller, 1997).

$$
C_{m} \frac{d V}{d t}=-I_{N a}-I_{K}-I_{C a}-I_{A}-I_{K C a}-I_{L}+I
$$

where $C_{m}$ is the specific membrane capacitance, $V$ is the membrane potential, and $I$ is the stimulus applied to the neuron. In this study, $I=I_{a m p} \sin (2 \pi f t)$ is used to mimic the external periodic input, where $I_{a m p}$ is the stimulus amplitude, $f$ is the stimulus frequency. To avoid the model neurons overly hyperpolarized, we set the negative values of $I$ to zero, the value under which the model neurons are quiescent.

For repetitive spiking RGC, $I_{\mathrm{Na}}=g_{\mathrm{Na}} m^{3} h\left(V-V_{\mathrm{Na}}\right)$, while for tonic and phasic RGC, $I_{N a}=g_{N a} m^{3} h s_{1} s_{2}\left(V-V_{N a}\right)$. The distinction between tonic and phasic RGCs is that they possess different combinational values of sodium channel conductance and potassium channel conductance (Wang et al., 2014).

Specific parameters used in our simulation are given in Table 1, and detailed expressions and gating variables for each ion current are provided in Table 2. In the table, $g_{N a}, g_{K}, g_{C a}, g_{A}$, $g_{K C a}$, and $g_{L}$ are the conductances for each currents. $V_{N a}, V_{K}$, and $V_{L}$ are the reversal potentials. $m, h, s_{1}, s_{2}, n, c, a$, and $b$ are the gating variables.

In contrast to sodium and potassium channels, which have constant values of reversal potential, the value of calcium channel is time-dependent (Equation 2).

$$
V_{C a}=\frac{R T}{Z F} \ln \left(\frac{\left[C a^{2+}\right]_{e}}{\left[C a^{2+}\right]_{i}(t)}\right)
$$

where $R$ is the gas constant, $T$ is the temperature in Kelvin, $Z$ is the ionic valency, $F$ is the Faraday constant, $\left[\mathrm{Ca}^{2+}\right]_{e}$ is the concentration of extracellular calcium, and the variation of intracellular calcium concentration $\left[\mathrm{Ca}^{2+}\right]_{i}$ obeys the Equation (3).

$$
\frac{d\left[\mathrm{Ca}^{2+}\right]_{i}}{d t}=\frac{-5 I_{C a}}{F r}-\frac{\left[\mathrm{Ca}^{2+}\right]_{i}-\left[\mathrm{Ca}^{2+}\right]_{r e s}}{\tau_{C a}}
$$

where $r$ is the depth of the shell beneath the membrane for the calcium pump, $\tau_{\mathrm{Ca}}$ is the time constant for calcium current, $\left[\mathrm{Ca}^{2+}\right]_{\text {res }}$ is the free intracellular concentration of calcium ions, and $\left[\mathrm{Ca}^{2+}\right]_{\text {diss }}$ is the calcium dissociation constants (see $I_{K C a}$ in Table 2).

\section{Morris-Lecar Model}

For comparison, the Morris-Lecar (ML) model was employed. It has been shown that the ML model can generate the three 
TABLE 1 | Specific parameters used in simulations.

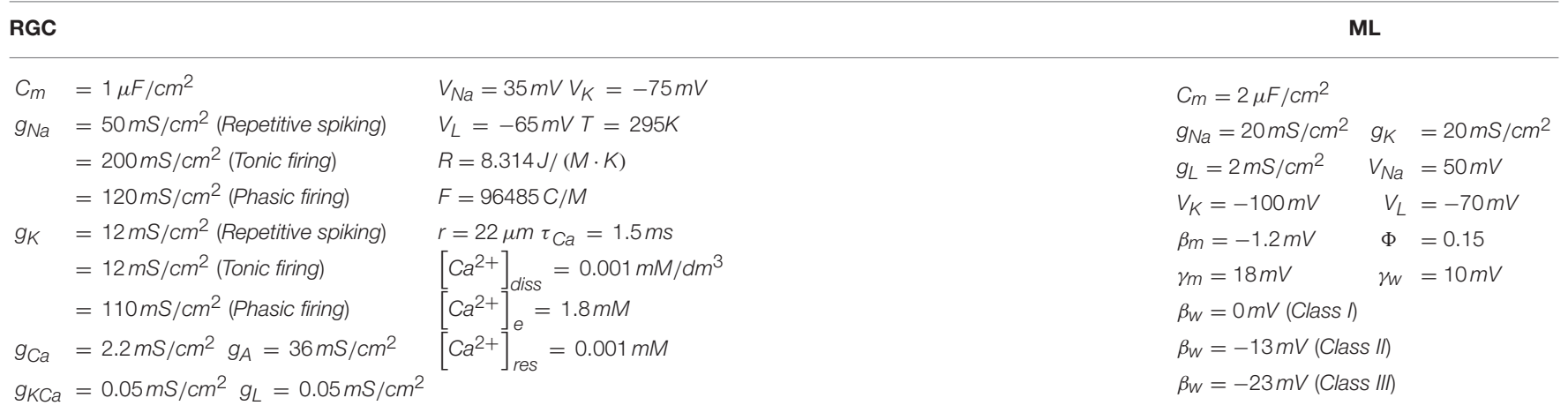

TABLE 2 | Specific expressions of ion currents and the corresponding gating variables.

\begin{tabular}{llll}
\hline Currents & & \multicolumn{1}{c}{ Gating variables } \\
\hline$I_{N a}=g_{N a} m^{3} h s_{1} S_{2}\left(V-V_{N a}\right)$ & $\begin{array}{l}\alpha_{m}=\frac{-0.1(V+30)}{\exp (-(V+30) / 10)-1} \\
\beta_{m}=4 \exp (-(V+55) / 18) \\
\alpha_{S_{2}}=0.0008 \exp (-V / 36)\end{array}$ & $\begin{array}{l}\beta_{h}=\frac{1}{1+\exp (-(V+20) / 10)} \\
\alpha_{h}=0.07 \exp (-(V+50) / 20)\end{array}$ & $\begin{array}{l}\beta_{S_{1}}=\frac{0.0014}{1+\exp (-(V+47) / 4.7)} \\
\alpha_{S_{1}}=0.00034 \exp (-V / 63)\end{array}$ \\
\hline$I_{K}=g_{K} n^{4}\left(V-V_{K}\right)$ & $\alpha_{n}=\frac{0.02(V+40)}{1-\exp (-(V+40) / 10)}$ & $\beta_{n}=0.4 \exp (-(V+50) / 80)$ \\
\hline$I_{C a}=g_{C a} C^{3}\left(V-V_{C a}\right)$ & $\alpha_{C}=\frac{0.3(V+13)}{1-\exp (-(V+13) / 10)}$ & $\beta_{C}=10 \exp (-(V+38) / 18)$ \\
\hline$I_{A}=g_{A} a^{3} b\left(V-V_{K}\right)$ & $\alpha_{a}=\frac{0.006(V+90)}{1-\exp (-(V+90) / 10)}$ & $\beta_{b}=\frac{0.6}{1+\exp (-(V+40) / 10)}$ \\
& $\alpha_{b}=0.04 \exp (-(V+70) / 20)$ & $\beta_{a}=0.1 \exp (-(V+30) / 10)$
\end{tabular}

$I_{K C a}=g_{K C a} \frac{\left(\left[\mathrm{Ca}^{2+}\right] /\left[\mathrm{Ca}^{2+}\right]_{\text {diss }}\right)^{2}}{1+\left(\left[\mathrm{Ca}^{2+}\right] /\left[\mathrm{Ca}^{2+}\right]_{\text {diss }}\right)^{2}}\left(V-V_{K}\right) \quad \quad I_{L}=g_{L}\left(V-V_{L}\right)$

In modeling the repetitive spiking behavior, $s_{1}$ and $s_{2}$ are both 1, same as in Fohlmeister and Miller (1997).

classes of excitability by only adjusting one parameter (Prescott et al., 2008a; Wang et al., 2013). Moreover, due to its simplicity and accuracy in mimicking the three classes of excitability, ML model has been widely used in analyzing the firing behavior of single neurons (St-Hilaire and Longtin, 2004; Prescott and Sejnowski, 2008; Wang et al., 2011) and population activity of neuronal networks (Marella and Ermentrout, 2008; Fink et al., 2011).

Mathematical expressions for the ML model are in Equations (4) and (5).

$$
\begin{aligned}
C_{m} \frac{d V}{d t}= & -g_{N a} m_{\infty}\left(V-V_{N a}\right)-g_{K} w\left(V-V_{K}\right) \\
& -g_{L}\left(V-V_{L}\right)+I \\
\frac{d w}{d t}= & \Phi\left(w_{\infty}-w\right) / \tau_{w}
\end{aligned}
$$

where $C_{m}$ is the specific membrane capacitance, $V$ is the membrane potential, $w$ is a slow recovery variable, and $I$ is the stimulus applied to the neuron which has the same form with that used in the RGC model. $g_{N a}, g_{K}$, and $g_{L}$ are the conductances for each currents. $V_{N a}, V_{K}$, and $V_{L}$ are the reversal potentials. $\Phi$ is a scaling factor.
In Equations (4) and (5), $m_{\infty}=0.5\left(1+\tanh \left(\left(V-\beta_{m}\right) / \gamma_{m}\right)\right)$, $w_{\infty}=0.5\left(1+\tanh \left(\left(V-\beta_{w}\right) / \gamma_{w}\right)\right), \tau_{w}=1 / \cosh ((V-$ $\left.\left.\beta_{w}\right) /\left(2 \gamma_{w}\right)\right)$

Other parameters used in simulations are shown in Table $\mathbf{1 .}$

It should be noted that the ML model used in this study is to generate typical features of the three classes of excitability, and compare to the performance of RGC model, the ML model itself cannot generate the repetitive spiking, tonic firing and phasic firing behaviors of RGCs.

Simulations of the RGC and ML models were performed in the MATLAB environment (R2010a), and the fourth-order RungeKutta algorithm was employed to calculate the voltage values of neurons with time integration being $0.01 \mathrm{~ms}$.

\section{RESULTS}

\section{Similar Performance between the Three Types of RGC and the ML Neurons}

Firstly, the repetitive spiking, tonic firing, and phasic firing behaviors of RGCs are simulated (Figure 1). It is clear that, the increase of stimulus intensity induces the repetitive spiking RGC switch from quiescent to fast spiking state, and the larger the stimulus intensity is, more spikes the neuron would elicit 


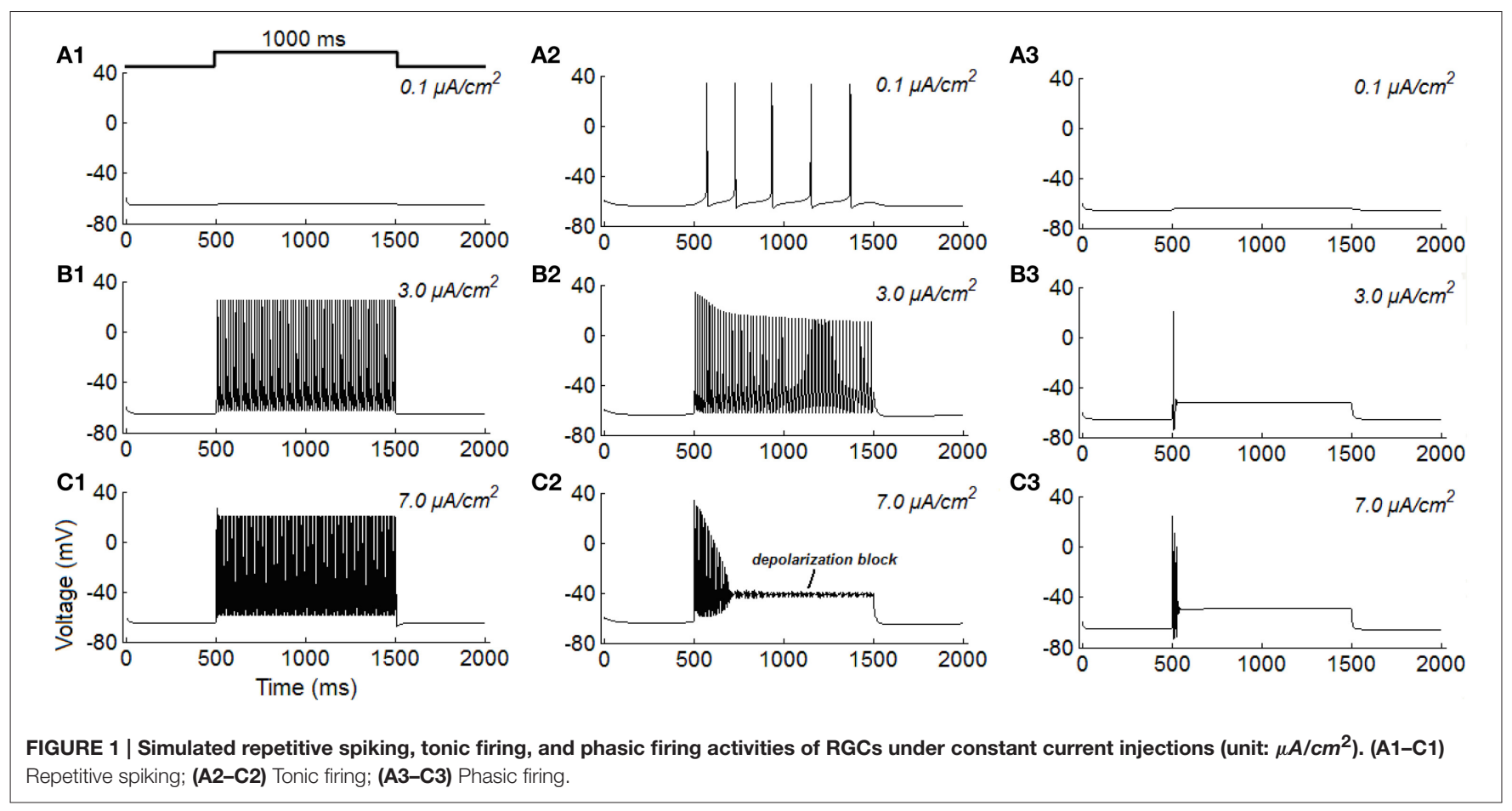

(Figures 1A1-C1), this variation trend is similar to that reported in Fohlmeister and Miller (1997), Fohlmeister et al. (2010) and Margolis and Detwiler (2007). For tonic RGC, it is found that the increase of stimulus intensity induces the neuron change from low-frequency firing state (Figure 1A2) to high-frequency firing state (Figure 1B2). However, when the stimulus intensity is too large, the neuron rapidly accommodates to a steady state in the late period of stimulation, a phenomenon called depolarization block (Figure 1C2). This variation trend is quite similar to previous experimental results (Kawai and Sterling, 2002; Tabata and Kano, 2002). While for phasic RGC, it is apparent that the increase of stimulus intensity makes the neuron transform from resting state (Figure 1A3) to spiking state at the onset of stimulus (Figures 1B3-C3). This variation trend is also similar to the experimental observations (Tabata and Kano, 2002; Kawai and Sterling, 2002).

In Figure 3A, it is clear that spike numbers of repetitive and phasic RGCs show similar monotonous increasing trends, with the spikes of repetitive spiking RGC being much more than those of the phasic RGC. However, spike numbers of tonic RGC demonstrate a non-monotonous variation trend: a highfrequency region is observed when the stimulus intensity locates in [1.5-5], while a low-frequency region is observed when the stimulus intensity locates in [6-8.5]. In the following analyses, we will separately discuss the response patterns of RGCs when stimulus intensity locates in these two different regions.

As observed in Figure 3A, the frequency of a repetitive spiking RGC varies in a discontinuous manner under weak stimulus, while the frequency of a tonic RGC varies in a continuous way. These variation trends are similar to that observed in Class I and Class II neurons. Moreover, the frequency of phasic RGC always stays in low states, which is also similar to the property of Class III neuron.

To make a better comparison, we simulate typical features of the three classes of excitability using the ML model. Results in Figure 2 are the typical firing behaviors of the three classes of neurons under different stimulus intensities. It is shown that the overall variations of firing activity of the ML neurons are comparable to the results observed in RGCs (Figure 1).

In Figure 3B, spike counts of the three neurons (ML) with respect to stimulus intensity are provided, which is clear that Class I neuron shows a continuous trend under weak stimulus, Class II neuron shows a discontinuous trend under weak stimulus, while Class III neuron always fires in low frequency states. Comparing Figure 3A with Figure 3B, we may conclude that repetitive spiking RGC exhibit Class II excitability, tonic RGC exhibit Class I excitability, and phasic RGC exhibit Class III excitability.

In addition to the spike counts, we also compare the spike latencies between the three RGCs and the three ML neurons. Results in Figure 4 demonstrate that RGCs exhibit much similar variation trends of latency with that of ML neurons, which further confirm that the three RGCs can exhibit Hodgkin's three classes of excitability.

\section{Amplitude-Dependent Firing Characteristics of RGCs under Periodic Stimuli}

Next, the capabilities of RGCs in encoding periodic signals are investigated. Two coding measures which have been widely used to characterize neural signals are employed, one measure is 

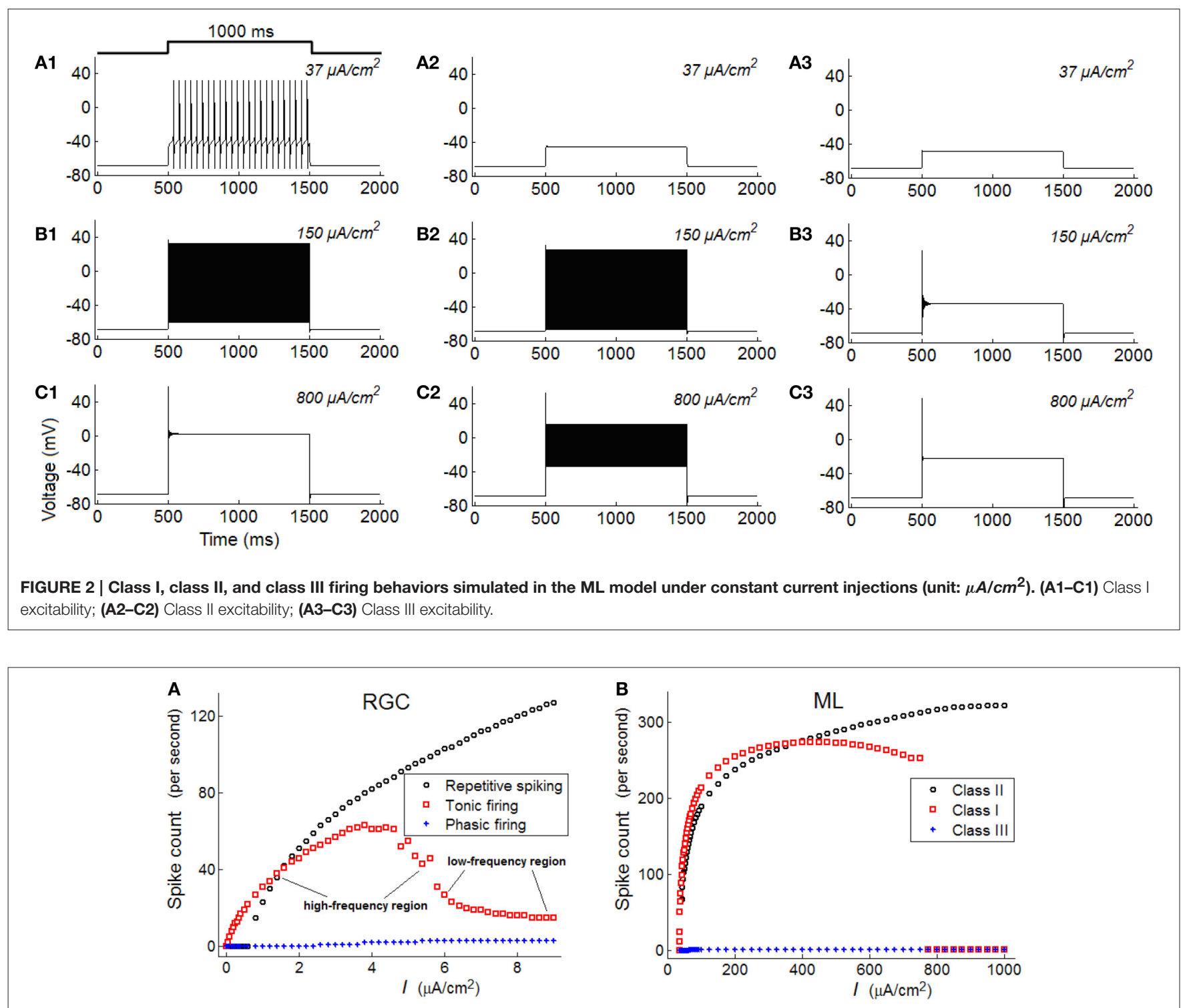

FIGURE 3 | Spike counts of the three types of RGC and the three classes of ML neurons with respect to stimulus intensity (unit: $\mu A / \mathrm{cm}^{2}$ ). (A) RGCs; (B) ML neurons.

the firing rate (spikes/cycle), which was chosen following the studies from van Brederode and Berger (2008) and Descalzo et al. (2005). We also did simulations using spikes/second to measure firing rate, and obtained similar comparison results between the RGC model and ML model (see Supplementary Figure); Another measure is the first-spike latency. The reason we adopt these two measures is that firing rates and first-spike latency have both found to effectively encode visual information in RGCs (van Rullen and Thorpe, 2001; Gollisch and Meister, 2008; Gütig et al., 2013).

As shown in Figure 5A1, under high $I_{a m p}$, there is an obvious region in which the firing rate of a repetitive spiking RGC with large values are located (dark red area), primarily under low stimulus frequencies. When low $I_{a m p}$ is applied, the phenomenon maintains, with only minor differences (Figure 5B1). These results suggest that repetitive spiking RGCs activate strongly under low stimulus frequencies, larger frequency would lower their firing activity. Moreover, the variation of $I_{a m p}$ barely alter this phenomenon. Figures 5A2-B2 demonstrates the results from a Class II neuron, which clearly show the similarity with those in a repetitive RGC.

Results in Figures 6A1-B1 are the case for a tonic RGC. It is clear that under high $I_{a m p}$, there is also an obvious region in which the firing rate with large values are located (dark red area in Figure 6A1), mainly under low stimulus frequencies. When low $I_{a m p}$ is applied, the observed feature persists (Figure 6B1), but a small distinction still can be seen. Under low $I_{a m p}$, the increase of stimulus amplitude would enhance the firing rate; while under high $I_{a m p}$, the increase of stimulus amplitude would weaken the firing rate, comparable with the result in Figure 3. These results indicate that although the variation of stimulus amplitude will not alter the general response patterns 

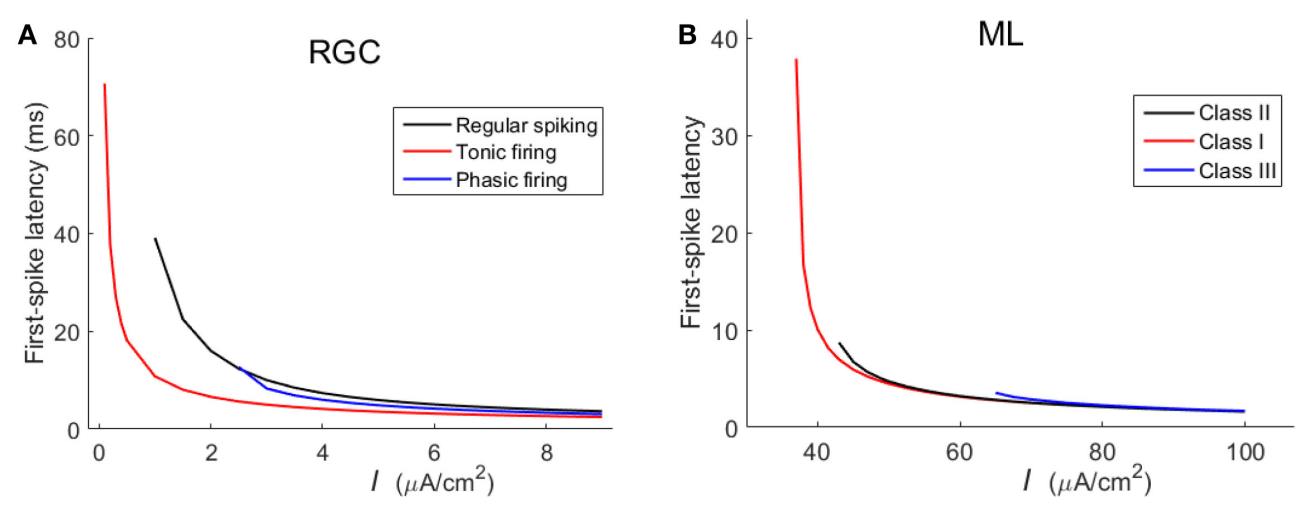

FIGURE 4 | First-spike latency of the three types of RGC and the three classes of ML neurons with respect to stimulus intensity (unit: $\mu A / \mathrm{cm}^{2}$ ). (A) RGCs; (B) ML neurons.
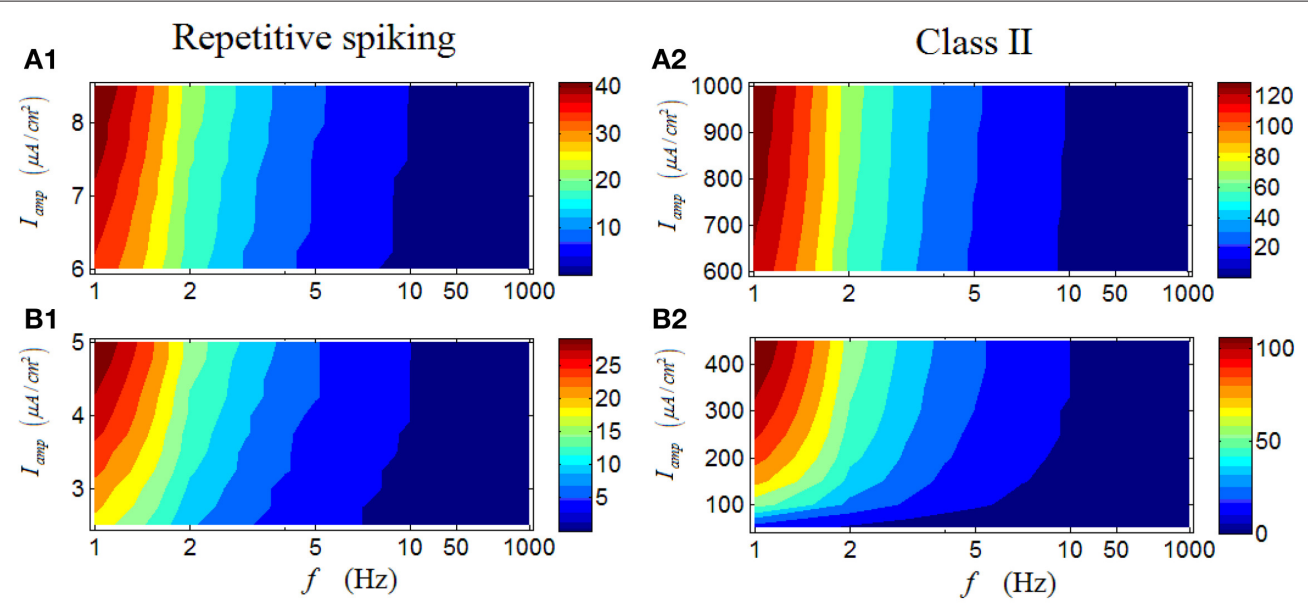

FIGURE 5 | Firing rates (spikes/cycle) of a repetitive spiking RGC and a Class II neuron under periodic stimuli. (A1,A2) Firing rates under high stimulus amplitude (lamp). (B1,B2) Firing rates under low stimulus amplitude (lamp).

of a tonic RGC, its detailed modulations are totally opposite when stimulus amplitude varies in the two different regions. Similar phenomenon can be observed in a Class I neuron (Figures 6A2-B2).

Results for a phasic RGC are illustrated in Figures 7A1-B1. It is apparent that the response characteristics are totally different between high and low $I_{a m p}$. Under low $I_{a m p}$, the phasic RGC responds vigorously at medium levels of stimulus frequency (Figure 7A1); while under high $I_{a m p}$, the phasic RGC switches to activate strongly at low stimulus frequencies (Figure 7B1). This group of result reveals that a phasic RGC can perform two different spike-rate coding operations, depending on the stimulus amplitude. Similar conclusion can be drawn in a Class III neuron (Figures 7A2-B2).

In the followings, we continue our study by analyzing the first-spike latency of RGCs. Results shown in Figures 8A1-B1 are the case for a repetitive spiking RGC, which indicate that the first-spike latency with large values are mostly located in a region with low stimulus frequencies (dark red area in Figures 8A1-B1). Meanwhile, variation of the stimulus amplitude has little influence on the general response pattern of the RGC. This result suggests that for a repetitive spiking RGC, relative high stimulus frequency would lead the neuron respond more rapidly, consistent with the result in Wang et al. (2013). Similar phenomenon can be observed in a Class II neuron (Figures 8A2-B2).

For a tonic RGC, its first-spike latency shows a similar variation trend with that of a repetitive spiking RGC (Figures 9A1-B1). However, unlike the results in Figure 6, in which the change of stimulus amplitude can markedly affect the firing rate of a tonic RGC, stimulus amplitude has little influence on the patterns of first- spike latency of the tonic RGC. Similar results can be produced by a Class I neuron (Figures 9A2-B2)

However, unlike the case for a repetitive spiking and a tonic RGC, the latency of a phasic RGC exhibits rather different variation trends. As demonstrated in Figure 10B1, a valley can be observed when $I_{a m p}$ is low, in which the latency decreases 

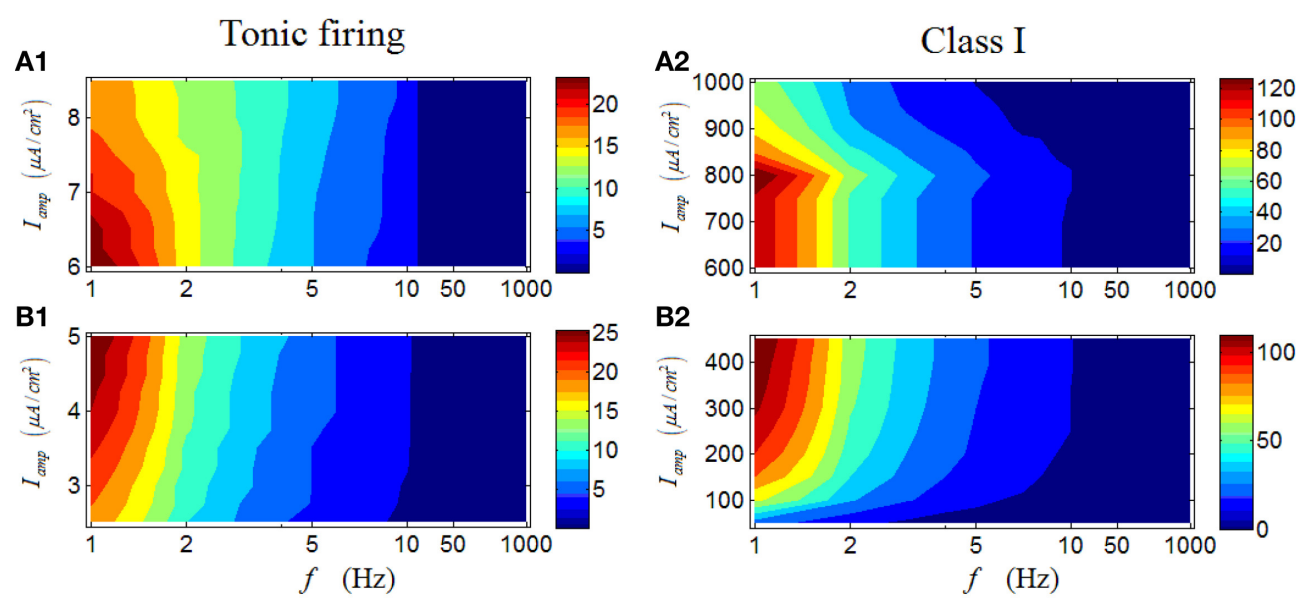

FIGURE 6 | Firing rates (spikes/cycle) of a tonic RGC and a Class I neuron under periodic stimuli. (A1,A2) Firing rates under high stimulus amplitude (lamp). (B1,B2) Firing rates under low stimulus amplitude (lamp).
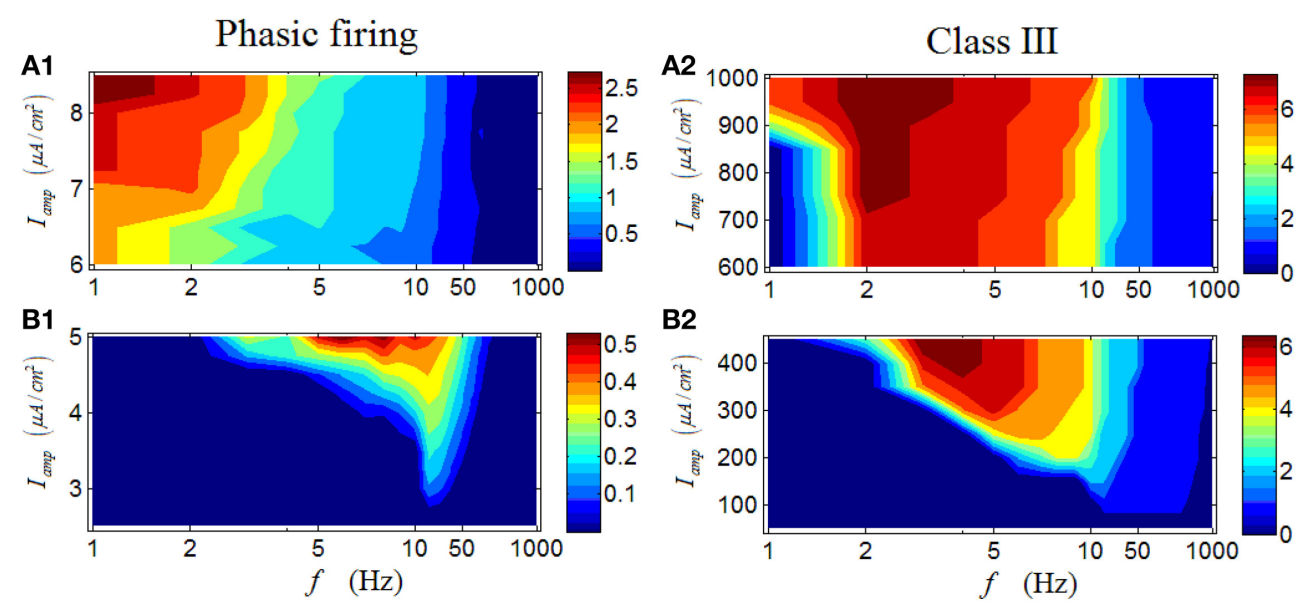

FIGURE 7 | Firing rates (spikes/cycle) of a phasic RGC and a Class III neuron under periodic stimuli. (A1,A2) Firing rates under high stimulus amplitude $\left(\right.$ lamp $_{\text {am }}$ (B1,B2) Firing rates under low stimulus amplitude $\left(l_{a m p}\right)$.

with the increase of stimulus frequency. When $I_{a m p}$ is high, the variation of latency with respect to stimulus frequency begins to change, specifically, the latency also decreases with the increased stimulus frequency but with a wider frequency range (Figure 10A1). In short, this group of results indicates that phasic RGC can perform a stable spike-latency coding pattern, and the change of stimulus amplitude would alter the response frequency range but would not alter this pattern significantly. A Class III neuron can exhibit similar variation trends (Figures 10A2-B2).

\section{DISCUSSIONS}

By revealing the exact mechanisms of various firing patterns in neurons, one can gain insight to different spiking behaviors and the nature of transition modes between them. This understanding is quite inspirational in promoting the exploration of how different factors might influence neural firing patterns. In this study, we performed a model investigation on the response properties of repetitive spiking, tonic firing, and phasic firing RGCs under periodic stimuli. Two coding measures (spike-rate and spike-latency) are employed to characterize the capability of the three RGCs in encoding periodic signals. The presented results suggest that repetitive spiking, tonic firing, and phasic firing RGCs can exhibit different firing characteristics in exposure to periodic stimuli. Meanwhile, our results further reveal that the dynamic response properties of RGCs are sensitive to the stimulus amplitude. More importantly, the results observed from the three RGCs can be well reproduced by Hodgkin's three classes of neuron (ML model).

As the first information processing center in the visual pathways, RGCs undertake important functions in encoding the incoming visual signals. However, a frequently encountered 

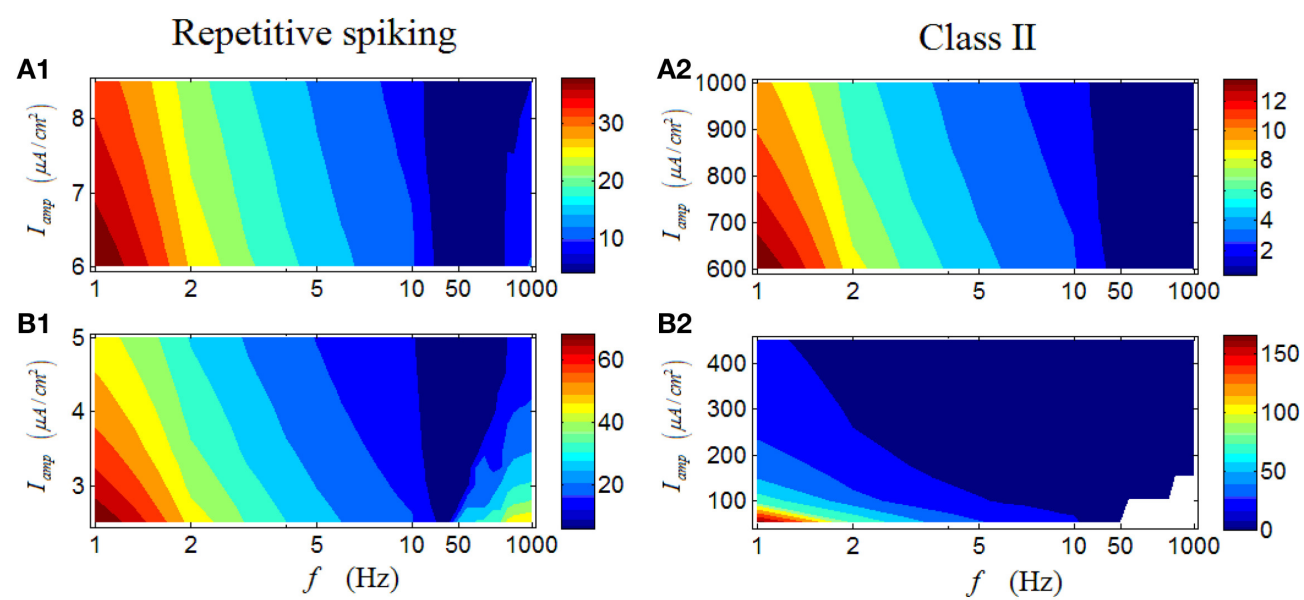

FIGURE 8 | First-spike latency of a repetitive spiking RGC and a Class II neuron under periodic stimuli. (A1,A2) First-spike latency under high stimulus amplitude (lamp). (B1,B2) First-spike latency under low stimulus amplitude (lamp). White area means there is no response of the neuron.
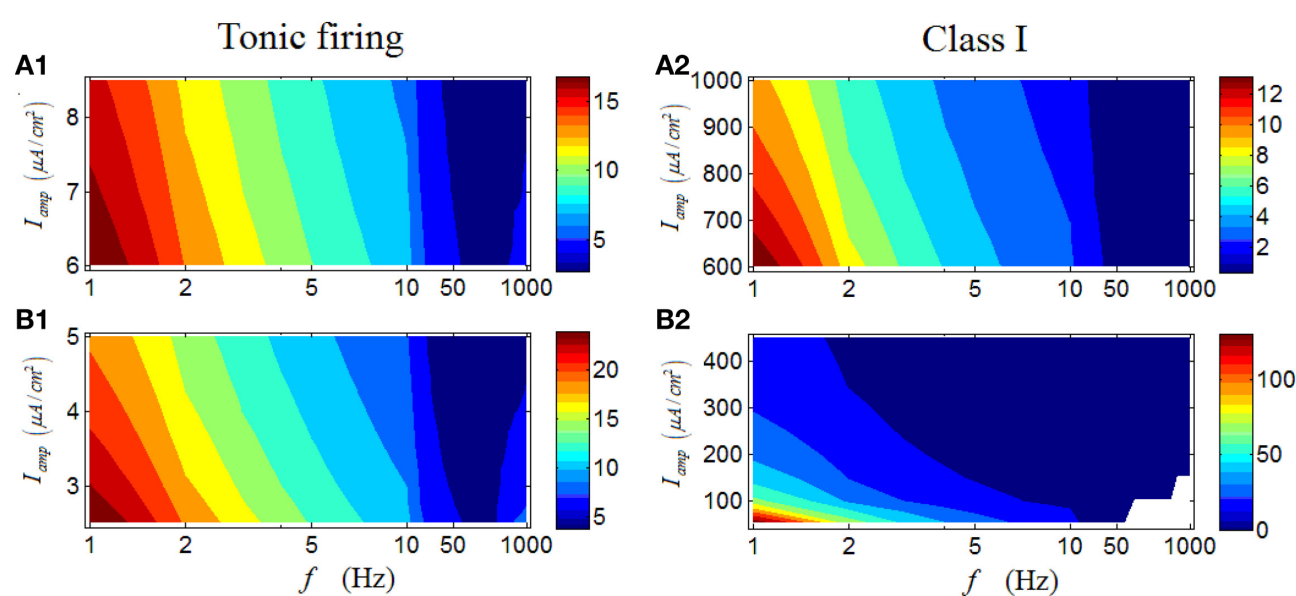

FIGURE 9 | First-spike latency of a tonic RGC and a Class I neuron under periodic stimulus. (A1,A2) First-spike latency under high stimulus amplitude (lamp). (B1,B2) First-spike latency under low stimulus amplitude $\left(I_{a m p}\right)$. White area means there is no response of the neuron.

problem for biological neurons is that their signaling capacity is too limited. Thus, to effectively encode the vast amount of visual inputs, RGCs must selectively react to some aspects of input signals, and ignore other aspects. Model results in this study suggest that different RGCs can perform different operations in encoding periodic signals. Repetitive spiking and tonic RGCs prefer to activate strongly under low-frequency stimulus, but respond weakly to high- frequency stimulus. On the other hand, phasic RGCs activate vigorously under medium levels of frequency, but respond sluggishly to lowand high-frequency stimuli. These distinctions may indicate that RGCs can expand their dynamic range through complementary actions. A similar phenomenon has been observed in frog second-order vestibular neurons $\left(2^{\circ} \mathrm{VN}\right)$ (Beraneck and Straka, 2011 ), in which the authors suggested that frog tonic $2^{\circ} \mathrm{VN}$ prefer to respond strongly to low-frequency stimulus, like low-pass filters, whereas phasic $2^{\circ} \mathrm{VN}$ prefer to respond strongly at medium levels of stimulus frequency, like bandpass filters.

The transformation of incoming signals into spikes by neurons is recognized to be the basis for neural information processing (neural coding). During the past decades, several efficient coding strategies have been proposed, such as: rate coding (Descalzo et al., 2005; van Brederode and Berger, 2008), and time coding (Mainen and Sejnowski, 1995; Chase and Young, 2007; Zhang et al., 2010). A recent study suggested that RGCs can encode input stimulus into both spike-rate and spike-latency simultaneously (Xiao et al., 2014). In this study, we further suggest that RGCs with intrinsically heterogeneities may encode periodic stimuli into different spike-rate patterns and spikelatency patterns; moreover, the patterns of spike-rate and spikelatency produced by the same RGCs are quite similar.

Excitability is an extraordinary feature in many biological neurons. Traditionally, neuronal excitabilities can be 

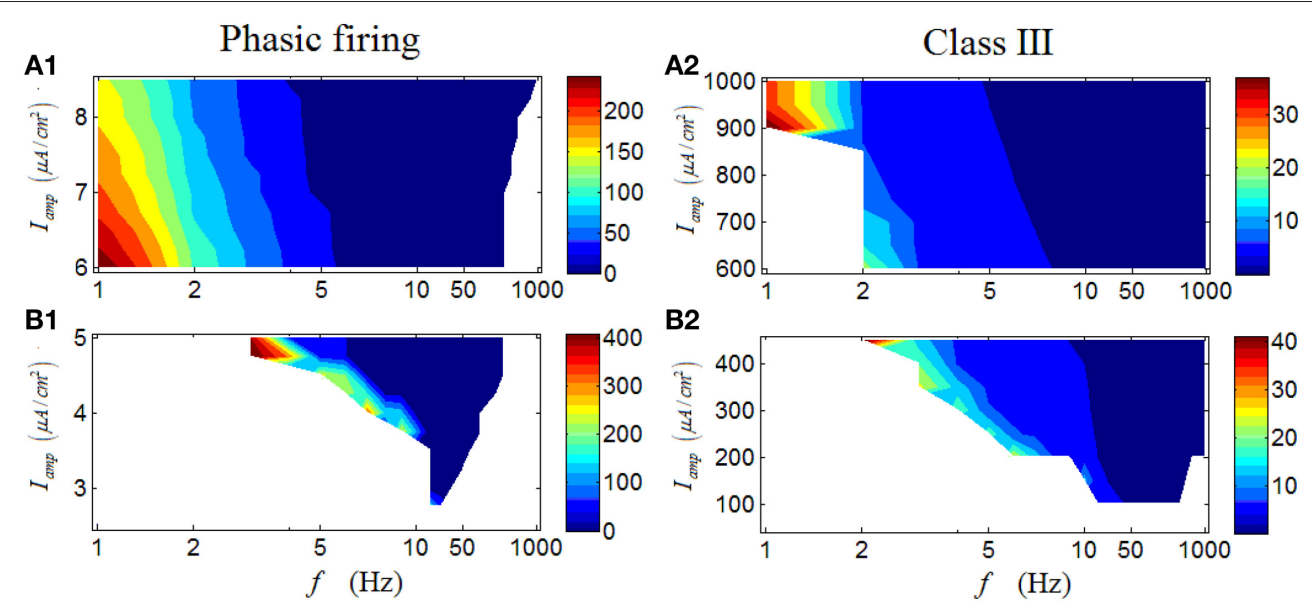

FIGURE 10 | First-spike latency of a phasic RGC and a Class III neuron under periodic stimuli. (A1,A2) First-spike latency under high stimulus amplitude $\left(l_{a m p}\right)$. (B1,B2) First-spike latency under low stimulus amplitude $\left(I_{a m p}\right)$. White area means there is no response of the neuron.

categorized into three basic classes (I, II, III) based on their electrophysiological responses to constant current injections (Hodgkin, 1948; Izhikevich, 2007). Many types of neurons have been found to exhibit part(s) or all of the three classes of excitability, for instance, some neurons were found to exhibit Class I or Class II firing dynamics (Tateno et al., 2004; Tateno and Robinson, 2006), or both Class I and II firing dynamics (Prescott et al., 2008b; Zeberg et al., 2010), while other neurons were observed to perform all the three classes of excitability (Prescott et al., 2008a). It has been reported that some ion channels exhibit crucial roles in distinguishing different classes of neurons, e.g., Na-K channel density (Johansson and Arhem, 1992; Arhem et al., 2006; Arhem and Blomberg, 2007), and M-type potassium current (Prescott et al., 2008b). In this model study, we suggest that RGCs can produce all the three classes of excitability by adjusting several ion channel densities. This is important, since RGCs play pivotal functions in encoding visual signals, to process the vast amount of light inputs efficiently, RGCs must adopt complementary strategies to expand their excitation range. Due to the universality of the three classes of excitability in neuronal systems, the results we observed from the RGCs model may be not specific, and we further infer that the results can applicable to other neurons in the brain regions which show part(s) or all of the three firing dynamics.

Previous reports have revealed that response patterns of periodically forced neurons can be modulated by a variety of factors, e.g., intrinsic ion channels (Cangiano et al., 2007; Kamiyama et al., 2009) and active dendrites (Zhuchkova et al., 2013). Besides, our results suggest that in RGCs, stimulus amplitude is also a critical factor in modulating the capability of RGCs in encoding periodic signals. In summary, model results in this study may provide new insights in further understanding how RGCs encode periodic afferents.
It should be noted that the model we analyze in this study is a simplified single-compartment neuron model with only one soma and did not include any dendritic information. During the past decades, a great number of studies adopt singlecompartment models as basis to simulate firing behavior of biological neurons and neuronal systems. However, it is still insufficient and inaccurate, since dendrites also play important roles in regulating neuronal activities. Thus, one extension of our study may concentrate on using more detailed models with active dendrites to simulate the firing behavior of RGCs using periodic forces. In addition, RGCs are more prone to activate collectively than activate independently, previous researches have suggested that gap junctions are abundant between RGCs, also between RGCs and other retinal cells (Völgyi et al., 2013). So, another extension of our study may concentrate on networks of RGCs coupled with gap junctions, and investigate how periodic forces would influence the network activity of RGCs.

\section{AUTHOR CONTRIBUTIONS}

LW and YQ designed and wrote this paper, LW collected and analyzed the data, YQ and YZ revised it and approved it.

\section{ACKNOWLEDGMENTS}

This work was supported by grants from Beijing Municipal Commission of Science and Technology (No. Z151100000915070).

\section{SUPPLEMENTARY MATERIAL}

The Supplementary Material for this article can be found online at: http://journal.frontiersin.org/article/10.3389/fncom. 2016.00102 


\section{REFERENCES}

Arhem, P., and Blomberg, C. (2007). Ion channel density and threshold dynamics of repetitive firing in a cortical neuron model. Biosystems 89, 117-125. doi: 10.1016/j.biosystems.2006.03.015

Arhem, P., Klement, G., and Blomberg, C. (2006). Channel density regulation of firing patterns in a cortical neuron model. Biophys J. 90, 4392-4404. doi: 10.1529/biophysj.105.077032

Beraneck, M., and Straka, H. (2011). Vestibular signal processing by separate sets of neuronal filters. J. Vestib. Res. 21, 5-19. doi: 10.3233/VES-201 1-0396

Bogaard, A., Parent, J., Zochowski, M., and Booth, V. (2009). Interaction of cellular and network mechanisms in spatiotemporal pattern formation in neuronal networks. J. Neurosci. 29, 1677-1687. doi: 10.1523/JNEUROSCI.5218-0 8.2009

Cangiano, L., Gargini, C., Della Santina, L., Demontis, G. C., and Cervetto, L. (2007). High-pass filtering of input signals by the Ih current in a non-spiking neuron, the retinal rod bipolar cell. PLoS ONE 2:e1327. doi: 10.1371/journal.pone.0001327

Chase, S. M., and Young, E. D. (2007). First-spike latency information in single neurons increases when referenced to population onset. Proc. Natl. Acad. Sci. U.S.A. 104, 5175-5180. doi: 10.1073/pnas.0610 368104

Dayan, P., and Abbott, L. F. (2001). Theoretical Neuroscience. Cambridge, MA: MIT Press.

Descalzo, V. F., Nowak, L. G., Brumberg, J. C., McCormick, D. A., and SanchezVives, M. V. (2005). Slow adaptation in fast-spiking neurons of visual cortex. J. Neurophysiol. 93, 1111-1118. doi: 10.1152/jn.00658.2004

Fink, C. G., Booth, V., and Zochowski, M. (2011). Cellularly-driven differences in network synchronization propensity are differentially modulated by firing frequency. PLoS Comput. Biol. 7:e1002062. doi: 10.1371/journal.pcbi. 1002062

Fohlmeister, J. F., Cohen, E. D., and Newman, E. A. (2010). Mechanisms and distribution of ion channels in retinal ganglion cells: using temperature as an independent variable. J. Neurophysiol. 103, 1357-1374. doi: 10.1152/jn.00123.2009

Fohlmeister, J. F., and Miller, R. F. (1997). Impulse encoding mechanisms of ganglion cells in the tiger salamander retina. J. Neurophysiol. 78, 1935-1947.

Gai, Y., Doiron, B., and Rinzel, J. (2010). Slope-based stochastic resonance: how noise enables phasic neurons to encode slow signals. PLoS Comput. Biol. 6:e1000825. doi: 10.1371/journal.pcbi.1000825

Gollisch, T., and Meister, M. (2008). Rapid neural coding in the retina with relative spike latencies. Science 319, 1108-1111. doi: 10.1126/science. 1149639

Gütig, R., Gollisch, T., Sompolinsky, H., and Meister, M. (2013). Computing complex visual features with retinal spike times. PLOS ONE 8:e53063. doi: 10.1371/journal.pone.0053063

Hodgkin, A. L. (1948). The local electric changes associated with repetitive action in a non-medullated axon. J. Physiol. 107, 165-181. doi: 10.1113/jphysiol.1948.sp004260

Izhikevich, E. (2000). Neural excitability, spiking and busting. Int. J. Bifurcat. Chaos 10, 1171-1266. doi: 10.1142/S0218127400000840

Izhikevich, E. M. (2007). Dynamical Systems in Neuroscience: The Geometry of Excitability and Bursting. Cambridge, MA: MIT Press.

Johansson, S., and Arhem, P. (1992). Membrane currents in small cultured rat hippocampal neurons: a voltage-clamp study. J. Physiol. 445, 141-156. doi: 10.1113/jphysiol.1992.sp018916

Kamiyama, Y., Wu, S. M., and Usui, S. (2009). Simulation analysis of bandpass filtering properties of a rod photoreceptor network. Vision Res. 49, 970-978. doi: 10.1016/j.visres.2009.03.003

Kandel, E. R., Schwartz, J. H., and Jessell, T. M. (2000). Principles of Neural Science. New York, NY: McGraw-Hill.

Kawai, F., and Sterling, P. (2002). cGMP modulates spike responses of retinal ganglion cells via a cGMP-gated current. Vis. Neurosci. 19, 373-380. doi: $10.1017 /$ S0952523802193138

Kim, K. J., and Rieke, F. (2003). Slow $\mathrm{Na}^{+}$inactivation and variance adaptation in salamander retinal ganglion cells. J. Neurosci. 23, 1506-1516.
Li, H., Liu, W. Z., and Liang, P. J. (2012). Adaptation-dependent synchronous activity contributes to receptive field size change of bullfrog retinal ganglion cell. PLOS ONE 7:e34336. doi: 10.1371/journal.pone.0 034336

Mainen, Z. F., and Sejnowski, T. J. (1995). Reliability of spike timing in neocortical neurons. Science 268, 1503-1506. doi: 10.1126/science.7770778

Marella, S., and Ermentrout, G. B. (2008). Class-II neurons display a higher degree of stochastic synchronization than class-I neurons. Phys. Rev. E 77:041918. doi: 10.1103/physreve.77.041918

Margolis, D. J., and Detwiler, P. B. (2007). Different mechanisms generate maintained activity in ON and OFF retinal ganglion cells. J. Neurosci. 27, 5994-6005. doi: 10.1523/JNEUROSCI.0130-07.2007

Prescott, S. A., De Koninck, Y., and Sejnowski, T. J. (2008a). Biophysical basis for three distinct dynamical mechanisms of action potential initiation. PLoS Comput. Biol. 4:31000198. doi: 10.1371/journal.pcbi.1000198

Prescott, S. A., Ratté, S., De Koninck, Y., and Sejnowski, T. J. (2008b). Pyramidal neurons switch from integrators in vitro to resonators under in vivo-like conditions. J. Neurophysiol. 100, 3030-3042. doi: 10.1152/jn.906 34.2008

Prescott, S. A., and Sejnowski, T. J. (2008). Spike-rate coding and spike-time coding are affected oppositely by different adaptation mechanisms. J. Neurosci. 28, 13649-13661. doi: 10.1523/JNEUROSCI.1792-08.2008

St-Hilaire, M., and Longtin, A. (2004). Comparison of coding capabilities of Type I and Type II neurons. J. Comput. Neurosci. 16, 299-313. doi: 10.1023/B:JCNS.0000025690.02886.93

Tabata, T., and Kano, M. (2002). Heterogeneous intrinsic firing properties of vertebrate retinal ganglion cells. J. Neurophysiol. 87, 30-41.

Tateno, T., Harsch, A., and Robinson, H. P. (2004). Threshold firing frequencycurrent relationships of neurons in rat somatosensory cortex: type 1 and type 2 dynamics. J. Neurophysiol. 92, 2283-2294. doi: 10.1152/jn.00109.2004

Tateno, T., and Robinson, H. P. (2006). Rate coding and spike-time variability in cortical neurons with two types of threshold dynamics. J. Neurophysiol. 95, 2650-2663. doi: 10.1152/jn.00683.2005

Tsai, D., Morley, J. W., Suaning, G. J., and Lovell, N. H. (2011). Frequencydependent reduction of voltage-gated sodium current modulates retinal ganglion cell response rate to electrical stimulation. J. Neural Eng. 8:066007. doi: 10.1088/1741-2560/8/6/066007

van Brederode, J. F., and Berger, A. J. (2008). Spike-firing resonance in hypoglossal motoneurons. J. Neurophysiol. 99, 2916-2928. doi: 10.1152/jn.01037.2007

van Rullen, R., and Thorpe, S. J. (2001). Rate coding versus temporal order coding: what the retinal ganglion cells tell the visual cortex. Neural Comput. 13, 1255-1283. doi: 10.1162/08997660152002852

Völgyi, B., Kovács-Oller, T., Atlasz, T., Wilhelm, M., and Gábriel, R. (2013). Gap junctional coupling in the vertebrate retina: variations on one thems? Prog. Retinal Eye Res. 34, 1-18. doi: 10.1016/j.preteyeres.2012.12.002

Wang, G. Y., Ratto, G., Bisti, S., and Chalupa, L. M. (1997). Functional development of intrinsic properties in ganglion cells of the mammalian retina. J. Neurophysiol. 78, 2895-2903.

Wang, H. T., Chen, Y. L., and Chen, Y. (2013). First-spike latency in Hodgkin's three classes of neurons. J. Theor. Biol. 328, 19-25. doi: 10.1016/j.jtbi.2013.03.003

Wang, H. T., Wang, L. F., Yu, L. C., and Chen, Y. (2011). Response of Morris-Lecar neurons to various stimuli. Phys. Rev. E 83:021915. doi: 10.1103/physreve.83. 021915

Wang, L., Liang, P. J., Zhang, P. M., and Qiu, Y. H. (2014). Ionic mechanisms underlying tonic and phasic firing behaviors in retinal ganglion cells: a model study. Channels 8, 298-307. doi: 10.4161/chan.28012

Xiao, L., Zhang, D. K., Li, Y. Q., Liang, P. J., and Wu, S. (2013a). Adaptive neural information processing with dynamical electrical synapses. Front. Comput. Neurosci. 7:36. doi: 10.3389/fncom.2013.00036

Xiao, L., Zhang, P. M., Wu, S., and Liang, P. J. (2014). Response dynamics of bullfrog ON-OFF RGCs to different stimulus durations. J. Comput. Neurosci. 37, 149-160. doi: 10.1007/s10827-013-0492-2

Xiao, L., Zhang, P. M., Xing, D., Liang, P. J., and Wu, S. (2013b). Shifted encoding strategy in retinal luminance adaptation: from firing rate to neural correlation. J. Neurophysiol. 110, 1793-1803. doi: 10.1152/jn.0022 1.2013 
Zeberg, H., Blomberg, C., and Arhem, P. (2010). Ion channel density regulates switches between regular and fast spiking in soma but not in axons. PLoS Comput. Biol. 6:e1000753. doi: 10.1371/journal.pcbi.1000753

Zhang, Y. Y., Jin, X., Gong, H. Q., and Liang, P. J. (2010). Temporal and spatial patterns of retinal ganglion cells in response to nature stimuli. Prog. Biochem. Biophys. 37, 389-396. doi: 10.3724/SP.J.1206.2009.00617

Zhuchkova, E., Remme, M. W., and Schreiber, S. (2013). Somatic versus dendritic resonance: differential filtering of inputs through non-uniform distributions of active conductances. PLoS ONE 8:e78908. doi: 10.1371/journal.pone. 0078908
Conflict of Interest Statement: The authors declare that the research was conducted in the absence of any commercial or financial relationships that could be construed as a potential conflict of interest.

Copyright (c) 2016 Wang, Qiu and Zeng. This is an open-access article distributed under the terms of the Creative Commons Attribution License (CC BY). The use, distribution or reproduction in other forums is permitted, provided the original author(s) or licensor are credited and that the original publication in this journal is cited, in accordance with accepted academic practice. No use, distribution or reproduction is permitted which does not comply with these terms. 